\title{
Hyperdense spleen after prolonged gold therapy
}

\author{
Tommaso Bartalena MD PhD, Maria Francesca Rinaldi MD
}

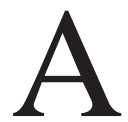

60-year-old woman underwent computed tomography imaging of her chest because of a recent episode of hemoptysis. The scan did not show any abnormalities in the thorax. However, unenhanced scans showed a strikingly hyperdense spleen (mean attenuation value 174 [normal 56-65] Hounsfield units) (Figure 1A). There were also hyperdense lymph nodes along the splenoportal axis and faint strands of increased density in the parenchyma of the peripheral liver. Imaging with contrast showed normal size and morphology of the organs with preserved contrast enhancement of the lymph nodes and spleen (Figure 1B).

Magnetic resonance imaging of the upper abdomen revealed normal signal intensity of spleen and liver both on $T_{1}$ - and $T_{2}$-weighted images, which ruled out hemochromatosis (Figure 1C). Ultrasonography showed normal splenic echotexture, thereby ruling out calcium deposition (e.g., may occur in autosplenectomy from sickle cell disease) (Figure 1D).

The patient had no history of receiving Thorotrast, a thorium-based angiographic contrast agent, or amiodarone therapy, but she had been given a prolonged course of intramuscular injections of sulfur colloidal gold for rheumatoid arthritis.

The computed tomography findings were consistent with deposition of gold particles in the reticuloendothelial system. Because of gold's high atomic number, deposition of gold can result in hyperdensity of spleen, liver and lymph nodes on computed tomography imaging. ${ }^{.}$Gold, unlike calcium, does not interfere with ultrasound transmission and, unlike iron, is a nonferromagnetic metal that does not alter the signal intensity of magnetic resonance imaging. Therefore, the ultrasonograph and

From the Department of Radiology (Bartalena), Poliambulatorio Privato Zappi Bartalena, Imola; and the Department of Radiology (Rinaldi), S. Orsola University Hospital, Bologna, Italy

CMAJ 2010. DOI:10.1503/cmaj.091962 magnetic resonance images were normal. However, the most important differential diagnosis of a hyperdense spleen is deposition of Thorotrast. This agent was discontinued after the 1950s because of its radioactivity and mutagen risk, which is associated with hepatosplenic malignancies. ${ }^{2}$ Gold compounds may mimic thorotrastosis at imaging, but their carcinogenic effect is negligible. ${ }^{3}$

This article has been peer reviewed.

Competing interests: None declared.

\section{REFERENCES}

1. De Maria M, De Simone G, Laconi A, et al. Gold storage in the liver: appearance on CT scans. Radiology 1986;159:355-6.

2. Eisler R. Chrysotherapy: a synoptic review. Inflamm Res 2003;52:487-501.

3. Levy DW, Rindsberg S, Friedman AC, et al. Thorotrast-induced hepatosplenic neoplasia: CT identification. AJR Am J Roentgenol 1988;146:997-1001. 